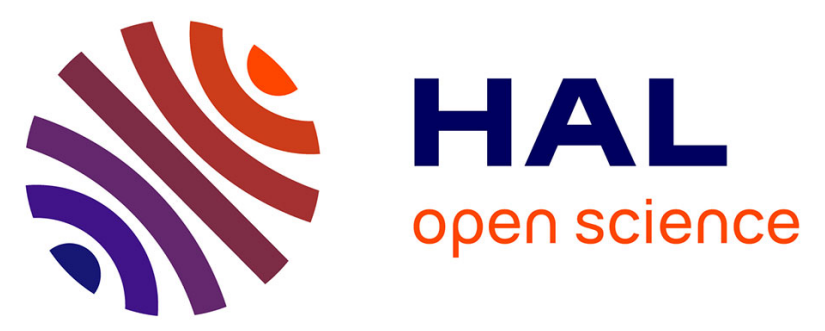

\title{
A Novel Adaptive Terminal Sliding Mode Control for Parallel Manipulators: Design and Real-Time Experiments
}

Moussab Bennehar, Gamal El-Ghazaly, Ahmed Chemori, François Pierrot

\section{To cite this version:}

Moussab Bennehar, Gamal El-Ghazaly, Ahmed Chemori, François Pierrot. A Novel Adaptive Terminal Sliding Mode Control for Parallel Manipulators: Design and Real-Time Experiments. ICRA: International Conference on Robotics and Automation, May 2017, Singapore, Singapore. pp.60866092, 10.1109/ICRA.2017.7989722 . lirmm-01718202

\section{HAL Id: lirmm-01718202 \\ https://hal-lirmm.ccsd.cnrs.fr/lirmm-01718202}

Submitted on 27 Feb 2018

HAL is a multi-disciplinary open access archive for the deposit and dissemination of scientific research documents, whether they are published or not. The documents may come from teaching and research institutions in France or abroad, or from public or private research centers.
L'archive ouverte pluridisciplinaire HAL, est destinée au dépôt et à la diffusion de documents scientifiques de niveau recherche, publiés ou non, émanant des établissements d'enseignement et de recherche français ou étrangers, des laboratoires publics ou privés. 


\title{
A Novel Adaptive Terminal Sliding Mode Control of PKMs: Design and Real-Time Experiments
}

\author{
Moussab Bennehar*, Gamal El-Ghazaly, Ahmed Chemori and François Pierrot
}

\begin{abstract}
This paper presents a new adaptive controller based on terminal sliding mode (TSM) control for parallel manipulators. More precisely, the proposed controller is based on a finite-time continuous TSM control scheme. To improve the tracking performance of parallel manipulators, a novel adaptive TSM control scheme is proposed in this paper. Based on the linear-in-the-parameters property of the dynamic model of rigid mechanical manipulators, an adaptive law is proposed to adjust the dynamic parameters of the manipulator in real-time. The proposed controller has the advantages of relying on the desired trajectories instead of measured ones which improves its robustness and efficiency. To demonstrate the effectiveness of the proposed controller, real-time experiments are conducted on a four degree-of-freedom (4-DOF) parallel manipulator called Veloce.
\end{abstract}

\section{INTRODUCTION}

Recent years have witnessed a rapidly increasing interest in parallel manipulators [1], [2]. This interest is driven by the fact that parallel manipulators possess various desirable properties in terms of high precision, high stiffness, dexterity, large velocity and acceleration capabilities compared to their serial counterparts [3]. These properties allow parallel manipulators to be adopted for a wide range of potential applications. Machine tools [4], pick-and-place [5], 3D printing [6], and haptic interfaces for robot-assisted surgery [7], [8] are just few of them. Parallel manipulators are closed chains of articulated rigid body systems. The development of precise dynamic models for these systems is known to be complex if not challenging. This is merely because a relatively large number of rigid bodies are involved for which the equations of motion have to be developed seen from an independent subset of joint coordinates, i.e. the actuated joints. To this end, the dynamics of the overall joint coordinates are projected onto the independent subset of coordinates [9]. Indeed, with this projection, the intricate structure of the inertial, Coriolis, and gravity terms in those independent coordinates do not only result in complex dynamic behavior but also require more real-time computations compared with serial manipulators. For these reasons, controller design of parallel manipulators is known to be challenging task [10]. With model complexity in mind, one may resort to simplified dynamics that captures only the dominant

*Corresponding author

LIRMM, Univ. Montpellier - CNRS, 161 rue Ada, 34095 Montpellier, France \{ bennehar, elghazaly, chemori, pierrot \} Qlirmm.fr inertial properties of the manipulator [11]. This is apart from the unknown joint frictions and the uncertainty in the inertial parameters that may arise for instance due to varying payloads. However, in order to handle the unmodeled dynamics from these simplifications, robust and/or adaptive control schemes are required to achieve high precision performances.

In the literature, several control schemes for parallel manipulators have been proposed [12], [10]. The earlier control schemes were based on the classical PD and PID controller [10]. However, these classical controllers usually provide unsatisfactory performance even at high control input torques and thus may be inadequate for applications requiring high tracking accuracy. In order to improve the overall performance of parallel manipulators, modern control strategies, mainly relying on the dynamic model of the manipulator in the control loop, were proposed [13]. Computed Torque [14] and Augmented PD [15] belong to this class. Nevertheless, to achieve the expected tracking performance, an accurate dynamic model of the manipulator is usually required. However, as explained earlier, obtaining an accurate dynamic model for parallel manipulators is not trivial and requires relatively more real-time computations. Model-based adaptive control [16] was proposed for parallel manipulators as a solution to overcome the problem of modelling uncertainties and inaccuracies, more precisely, the structural uncertainties (also called parametric uncertainties) in which the unknown parameters appear linearly in the dynamic model [17]. However, it is not always straightforward to rewrite the unmodeled dynamics resulting from simplified dynamic models of a parallel manipulator in a parametric form. To handle these nonparametric uncertainties, robust control schemes may be adopted. Robust control approaches that are based on sliding modes can effectively deal with such nonparametric but bounded uncertainties where the variable structure of the control law is designed to enforce the states of the system to stay in a chosen switching manifold defining the desired performance [18]. One major issue of classical sliding mode control schemes is that high chattering and saturation on the control input torques may arise due the higher bounds on uncertainties [18]. If equipped with an adaptation mechanism for parametric uncertainties, sliding mode control may result relatively lower chattering due to the reduction in the unknown uncertainty bounds. From this view point, combined robust and adaptive control schemes are therefore naturally the appropriate 
choice to simultaneously deal with both types of uncertainties in parallel manipulators and hence a better performance would be achieved.

The choice of switching manifold in sliding mode control greatly influences the dynamic performance of sliding mode control. Linear hyperplanes as in classical sliding mode guarantee asymptotic stability i.e., the closed-loop error converges to the neigh borhood of the origin as the time approaches infinity. The performance of classical sliding mode control could be enhanced if the closed-loop errors are forced to reach the origin in finite time. Sliding modes with finite-time convergence are called terminal sliding mode (TSM). Several ideas of designing TSM based control schemes have been developed to achieve finite-time stabilization in [19], [20], [21], [22]. A robust TSM control for robotic manipulators has been developed for robotics manipulators in [19]. Nevertheless, the main issue is that the controller may have a singularity problem in sliding mode. To avoid this controller singularity problem, a different terminal sliding mode used in [20], [21]. To improve the performance, TSM is equipped with an adaptive law in [23] to estimate the bounds of Coriolis, centrifugal and gravity terms that are approximated by polynomials of first order. An attempt to design an adaptive TSM control that estimates the true inertial parameters of a cable-driven parallel manipulator appears in [24]. However, the proposed adaptive law may have an initialisation problem if the initial state of the robot is the same as that of the desired trajectory.

The proposed adaptive TSM control scheme have the following advantages. First, it avoids the singularity problem that appeared in [19]. The problem of high chattering in the control input appeared in [20] is treated thanks to the choice of the continuous terminal sliding mode proposed in [21]. The proposed control scheme of this paper does not rely on the existence of a known nominal part of the manipulator dynamic model as in [21]. Compared with the adaptive TSM proposed in [23], some physically meaningful inertial parameters instead of the coefficients of polynomial functions bounding Coriolis and gravity terms so as to have better convergence of estimated parameters and consequently better an overall performance. The adaptive law of the proposed TSM control scheme is modified such that not only to avoid the initialisation problem appeared in [24] but also drive the estimated parameters to converge in finite time. The proposed control scheme is experimentally validated on a 4-DOF parallel manipulator, developed in our laboratory, called Veloce.

The paper is organized as follows. Section II describes the kinematic and the simplified dynamic modeling of Veloce. Section III is dedicated to the synthesis and design of the proposed adaptive terminal sliding mode control scheme for parallel manipulators. The experimental validations to show improved performance of the proposed control scheme is presented in Section IV. Section V concludes this paper.

\section{DESCRIPTION AND MODELING OF VELOCE PARALLEL ROBOT}

\section{A. Description of Veloce}

The Veloce robot, whose CAD is shown in Fig. 1, is a $3 \mathrm{~T} 1 \mathrm{R}$ parallel manipulator which means that the motion pattern of the platform is three dependent translations and one rotation. This pattern is widely known as Schönflies motion. Manipulators giving this motion pattern are called Schönflies motion (SMG) which are mainly intended for pick-and-place applications. Veloce is a Delta-like [25] parallel manipulator, with the peculiarity of having four kinematic chains instead of three. Each kinematic chain is a serial arrangement of an actuator, a rear-arm and a lightweight forearm. Moreover, the platform of Veloce is mainly composed of two parts (i.e. an upper part and a lower part). Each two opposite kineamtic chains are responsible for the motion of part of the platform. The relative motion of one part of the moving platform with respect to the other part results in the rotation of the platform.

\section{B. Kinematics}

Since Veloce is a $3 \mathrm{~T} 1 \mathrm{R}$ parallel manipulator, the pose of the platform can be parametrized through a 4dimensional coordinate vector $X=[x, y, z, \alpha]^{T}$. Let $q_{i}$ denotes angular position of actuator $i, \forall i=1,2, \ldots, 4$. Since Veloce is fully actuated, the actuator positions $q=\left[q_{1}, q_{4}, q_{3}, q_{4}\right]^{T}$ can completely specify the configuration of the entire mechanism. The relation between the configuration vector $q$ and platform pose $x$, known as the geometric model, is determined through the geometric constraints formed by the loop closure between each leg and the platform. For leg $i$, the geometric constraint

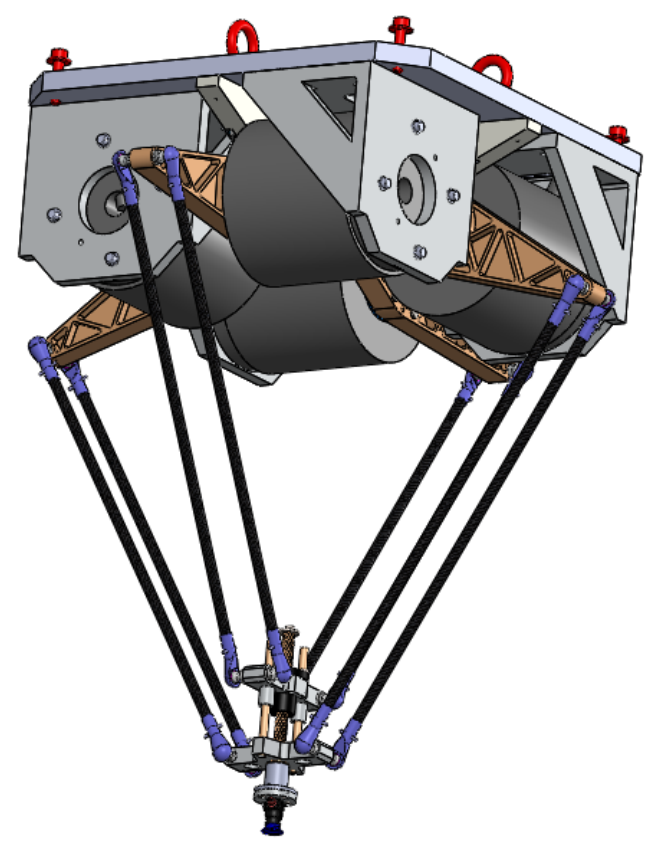

Fig. 1. CAD model of VELOCE robot 
could be chosen, for instance, such that length of the forearm is maintained constant, i.e.

$$
\left\|p_{B_{i}}-p_{C_{i}}\right\|^{2}-L^{2}=0, \forall i=1,2, \ldots, 4
$$

where $p_{B_{i}}$ and $p_{C_{i}}$ are the position coordinate vectors of the points $B_{i}$ and $C_{i}$, respectively, expressed in a fixed frame attached to the base of the robot and $L$ is the length of the forearm. The coordinates of $p_{B_{i}}$ are computed using $q_{i}$ while the coordinates of $p_{C_{i}}$ are computed using the platform pose $X$. Stacking the geometric constraint (1) $\forall i=1,2, \ldots, 4$, we get

$$
f(q, X)=0 .
$$

The differentiation of (2) with respect to yields the following kinematic constraints

$$
J_{q} \dot{q}+J_{x} \dot{X}=0
$$

where $J_{q}$ and $J_{x}$ are $4 \times 4$ Jacobian matrices. Note that these matrices are full rank if the trajectory is chosen to be free of singularity. Therefore, one may rewrite (3) as

$$
\dot{X}=J \dot{q},
$$

where $J=-J_{x}^{-1} J_{q}$

\section{Dynamics}

In this paper, the proposed control scheme involves the use of the dynamic model of the manipulator in the control design. Consequently, a brief description of the simplified dynamic model of VELOCE is presented in the sequel. But first, to simplify the motion equations of the different parts of the mechanical structure of VELOCE,

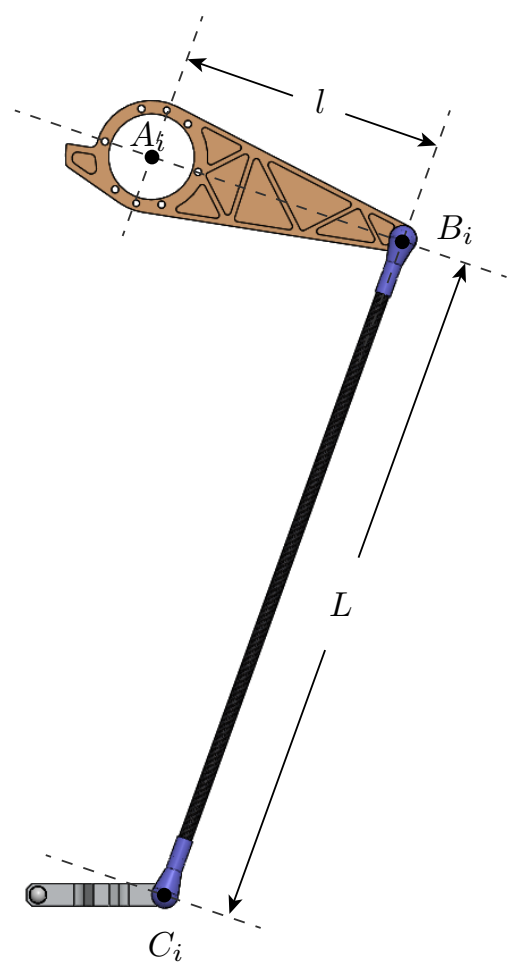

the following assumptions, commonly used in Delta-like robots, are considered [26]

Assumption 1: Both dry and viscous frictions in all passive and active joints are neglected. This is mainly due to the fact that the joints are carefully designed such that friction effects are minimized.

Assumption 2: The rotational inertia of the forearms is neglected and their mass is split up into two equivalent parts, one is added to the mass of the arm while the other one is considered with the moving platform. This hypothesis is justified by the small mass of the forearms compared to other components.

The dynamics of the VELOCE robot shows a lot of similarities with those of the Delta robot. Nevertheless few differences arise due to the number of kinematic chains and the additional rotational DOF of the moving platform.

Regarding the moving platform, we distinguish two kinds of forces acting on it, the gravity forces $G_{p} \in \mathbb{R}^{4}$ and the inertial force $F_{p} \in \mathbb{R}^{4}$, they are given by

$$
\begin{aligned}
G_{p} & =M_{p}\left[\begin{array}{llll}
0 & 0 & -g & 0
\end{array}\right]^{T} \\
F_{p} & =M_{p} \ddot{X}
\end{aligned}
$$

where $M_{p} \in \mathbb{R}^{4 \times 4}$ is the mass matrix of the moving platform that also considers the half-masses of the forearms, $g=9.81 \mathrm{~m} / \mathrm{s}^{2}$ is the gravity constant and $\ddot{X} \in \mathbb{R}^{4}$ is the Cartesian acceleration vector.

The contributions of $G_{p}$ and $F_{p}$ to each motor can be computed using the Jacobian matrix $J(q, X) \in \mathbb{R}^{4 \times 4}$ as follows

$$
\begin{aligned}
\Gamma_{G_{p}} & =J^{T} M_{p}\left[\begin{array}{llll}
0 & 0 & -g & 0
\end{array}\right]^{T} \\
\Gamma_{p} & =J^{T} M_{p} \ddot{X}
\end{aligned}
$$

From the joints side, the elements that contribute to the dynamics of the actuators are the forces and torques resulting from the movement of the rear-arms in addition to the half-messes of the forearms.

Applying the virtual work principle, which states that the sum of non-inertial forces is equal to that of the inertial ones, and after rearranging the terms, we get

$$
J^{T} M_{p} \ddot{X}+\Gamma_{G_{p}}+I_{a} \ddot{q}+\Gamma_{G_{a}}=\Gamma
$$

being $I_{a} \in \mathbb{R}^{4 \times 4}$ a diagonal inertia matrix of the arms accounting for the rear-arms as well as the half-masses of the forearms, $\ddot{q} \in \mathbb{R}^{4}$ the joint acceleration vector and

TABLE I

Main parameters of Veloce robot

\begin{tabular}{|c|c|c|}
\hline Parameter & Description & Value \\
\hline$l$ & Rear arms length & $0.2 \mathrm{~m}$ \\
$m_{r a}$ & Rear arms mass & $0.541 \mathrm{~kg}$ \\
$L$ & Forearms length & $0.53 \mathrm{~m}$ \\
$m_{f a}$ & Forearms mass & $0.08 \mathrm{~kg}$ \\
$I_{a c}$ & Actuators' inertia & $0.0041 \mathrm{~kg} \cdot \mathrm{m}^{2}$ \\
$m_{p}$ & Platform's mass & $0.999 \mathrm{~kg}$ \\
\hline
\end{tabular}


$\Gamma_{G_{a}} \in \mathbb{R}^{4}$ is the force vector resulting from gravity acting on the arms being given by

$$
\Gamma_{G_{a}}=m_{a} r_{G_{a}} g \cos (q)
$$

with $m_{a}$ the sum of the mass of one rear-arm and one half-mass of a forearm, $r_{G_{a}}$ the distance between the center of one axis and the center of mass of one arm and $q \triangleq\left[q_{1} q_{2} q_{3} q_{4}\right]^{T}$ is the actuated joints position vector.

Given the kinematic relationship $\ddot{X}=J \ddot{q}+\dot{J} \dot{q}$, (9) can be rewritten as follows

$$
\begin{aligned}
\left(I_{a}+J^{T} M_{p} J\right) \ddot{q}+ & \left(J^{T} M_{p} \dot{J}\right) \dot{q} \\
& -\left(J^{T} G_{p}+\Gamma_{G_{a}}\right)=\Gamma,
\end{aligned}
$$

which can be written in a standard joint-space form as follows

$$
M(q) \ddot{q}+C(q, \dot{q}) \dot{q}+G(q)+\Gamma_{d}=\Gamma
$$

with:

- $M(q)=I_{a}+J^{T} M_{p} J$ the total mass matrix.

- $C(q, \dot{q})=J^{T} M_{p} \dot{J}$ the Coriolis and centrifugal forces matrix.

- $G(q)=-\left(J^{T} G_{p}+\Gamma_{G_{a}}\right)$ the gravitational forces vector.

- The additional term $\Gamma_{d}$ stands for possible disturbances and inaccuracies in the dynamic model.

The dynamic model of Veloce in (12) is suitable for joint-space control since it is expressed in terms of the actuated joints. Being a rigid manipulator, the left hand side of (11) can be rewritten as follows [17]

$$
Y(q, \dot{q}, \ddot{q}) \Theta=\Gamma,
$$

where $Y(q, \dot{q}, \ddot{q}) \in \mathbb{R}^{4 \times p}$ is called the regression matrix, $\Theta \in \mathbb{R}^{p}$ is a constant vector of dynamic parameters and $p$ is the number of these parameters.

\section{ADAPTIVE TSM CONTROL DESIGN}

In this section, we develop an adaptive TSM control scheme for parallel manipulators. The notion behind TSM is that it allows reaching the sliding manifold and convergence to equilibrium both in finite time. Thus, control designs based on TSM are supposed to be more robust and with high performance. In addition to that, adaptive control may render enhanced performance if equipped with a TSM making it possible to meet the requirements of those applications in needed of high precision as in parallel manipulators.

Consider the desired position, velocity and acceleration trajectories of the moving platform be defined by $X_{d}, \dot{X}_{d}, \ddot{X}_{d}$, respectively. The desired actuators' position, velocity and acceleration trajectories, denoted by $q_{d}, \dot{q}, \ddot{q}_{d}$ respectively, can be obtained by using the adequate kinematic relationships. Let $e \triangleq q-q_{d}$ denote the position error of the actuated joints. In standard terminal sliding mode control, the following sliding manifold is defined [19]

$$
s=\dot{e}+\beta e^{p / q},
$$

where $\beta>0$ is a design parameter and $p>q>0$ are odd integers. In order to satisfy the reachability condition $(1 / 2)(d / d t) s^{T} s<-\eta|s|, \quad \eta>0$, the following control law can be chosen [20]

$$
\Gamma=Y(q, \dot{q}, v) \Theta,
$$

where $\rho=\lambda_{m} \zeta$ and the auxiliary control term $v$ is given by

$$
v \triangleq \ddot{q}_{d}+\beta \frac{q}{p} e^{p / q-1} \dot{e}+(\rho+\eta \operatorname{sign}(s))
$$

The control law of standard TSM in (15) considers that the dynamic parameters of the parallel manipulator $\theta$ are known and the bound on the disturbance term $\Gamma_{d}$ is known. Nevertheless, the control law (15) may suffer from singularity issues and is discontinuous which may lead to chattering [20].

To avoid the problem of singularity of (15), the following sliding manifold can be chosen instead [21]

$$
s=e+\beta|\dot{e}|^{\gamma} \operatorname{sign}(e),
$$

where $\beta>0$ and $1<\gamma<2$ are control design parameters. The sliding manifold (17) has the advantages of being continuous in time. Its first time derivative is given by $[21] \dot{s}=\dot{e}+\beta \gamma|\dot{e}|^{\gamma-1} \ddot{e}$. Moreover, the terminal sliding mode (17) has a global finite-time equilibrium $t_{r}$ given by [21]

$$
t_{r} \leq \frac{\gamma \beta^{1 / \gamma}}{\gamma-1}|e(0)|^{(\gamma-1) / \gamma}
$$

If the dynamic parameters of the parallel manipulator are perfectly known and in the absence of uncertainties (i. e. $\Gamma_{d}=0$ ), the following control law can be chosen in order to ensure that $e$ converges to zero in finite-time

$$
\Gamma=Y(q, \dot{q}, v) \Theta-K_{1} s-K_{2}|s|^{\rho} \operatorname{sign}(s)
$$

where $K_{1}$ and $K_{2}$ are positive definite design diagonal matrices, $\rho<1$ and the auxiliary control term $v$ is defined by

$$
v \triangleq \ddot{q}_{d}-\frac{1}{\beta \gamma}|\dot{e}|^{2-\gamma} \operatorname{sign}(\dot{e})
$$

The control law (19) considers the dynamic model to be accurately known. However, in practice, the dynamic parameters of parallel manipulators could be unknown, uncertain or time-varying (e. g. changing payload). Even though those variations will be handled as any general disturbance term, the fact that their structure is supposed unknown can lead to poor tracking performance, high feedback gain and chattering.

Adaptive controllers are known to be the most adequate solution to handle uncertainties with known structure. Consequently, in order to benefit from both adaptive control and terminal sliding mode, the best solution is to combine both terminal sliding mode and adaptive control in a single controller.

The main contribution of this paper is to develop a TSM-based adaptive controller. The motivation behind such idea is to take advantage from the robustness of 
TSM control while obtaining improved tracking thanks to the real-time adaptation of the dynamic-based control loop. To that aim, consider the following control law

$$
\Gamma=Y\left(q_{d}, \dot{q}_{d}, \ddot{q}_{d}\right) \hat{\Theta}(t)-K_{1} s-K_{2}|s|^{\rho} \operatorname{sign}(s),
$$

where $K_{1}$ and $K_{2}$ were defined in (19).

The estimated parameters' vector $\hat{\Theta}(t)$ is adjusted in real-time according to the following adaptation law

$$
\dot{\hat{\Theta}}(t)=-\Xi Y\left(q_{d}, \dot{q}_{d}, \ddot{q}_{d}\right)^{T}\left(K_{3} s+K_{4}|s|^{\rho} \operatorname{sign}(s)\right),
$$

where $\Xi \in \mathbb{R}^{p}$ is a positive definite diagonal matrix known as the adaptation gain matrix and $K_{3}$ and $K_{4}$ are diagonal positive definite design gain matrices.

As it can be seen, the proposed control law (22) has the advantage of being simple to implement since it mainly relies on the desired trajectories. Indeed, the regression matrix in (22) is evaluated using the desired actuators' trajectories $q_{d}, \dot{q}_{d}$ and $\ddot{q}_{d}$. Unlike measured ones, desired trajectories can be computed and stored offline which significantly reduces the computation cost of the controller. Moreover, desired trajectories are immune to measurement noise, which means that the robustness of the controller is enhanced. Similarly, the adaptation law inherits the previous advantages since it is also based on desired trajectories.

\section{EXPERIMENTAL RESULTS}

The actuators of VELOCE are the TMB0140100-3RBS ETEL direct-drive motors. They can provide a maximum peak torque of $127 \mathrm{Nm}$ and are able to reach $550 \mathrm{rpm}$ of speed. Each actuator is equipped with a non-contact incremental optical encoder providing a total number of 5000 pulses per revolution. The global structure of the manipulator is able to reach $10 \mathrm{~m} / \mathrm{s}$ of maximum velocity of the traveling plate, $200 \mathrm{~m} / \mathrm{s}^{2}$ of

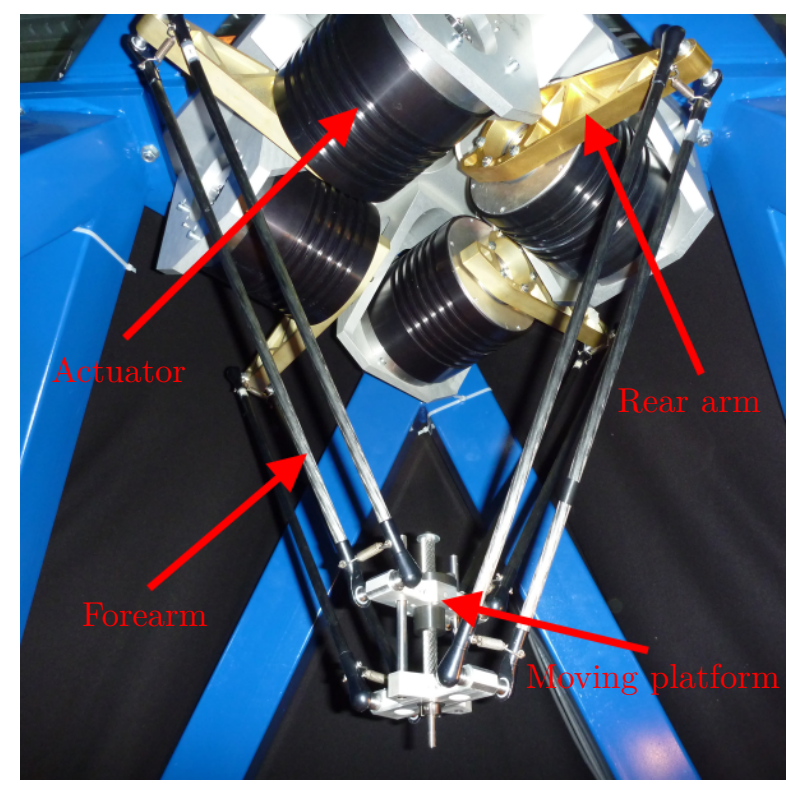

Fig. 2. View of the experimental setup of the VELOCE robot.
TABLE II

SumMARY ON CONTROL DESIGN PARAMETERS

\begin{tabular}{|c|c|c|c|}
\hline Parameter & Value & Parameter & Value \\
\hline$\beta$ & 0.004 & $K_{2}$ & $35 \times I_{4}$ \\
$\gamma$ & 1.25 & $\Xi$ & $\operatorname{diag}([1.5,0.015])$ \\
$\rho$ & 0.75 & $K_{3}$ & $20 \times I_{4}$ \\
$K_{1}$ & $1000 \times I_{4}$ & $K_{4}$ & $I_{4}$ \\
\hline
\end{tabular}

maximum acceleration and is able to handle a maximum payload of $10 \mathrm{Kg}$. The control architecture of the VELOCE robot is implemented using Simulink from from Mathworks Inc. and compiled using XPC Target and the Real-Time toolboxes. The resulting low-level code is then uploaded to the target PC; an industrial computer cadenced at $10 \mathrm{KHz}$ (i.e. sample time of $0.1 \mathrm{~ms}$ ). The experimental testbed is displayed in Fig. 2.

To highlight the benefits of the proposed adaptive controller, both the standard TSM and the proposed controllers were implemented on Veloce robot. The parameters of both controllers are summarized in Table IV. In order to quantify the control performance, we introduce the following criteria based on the Root Mean Square of the tracking Error (RMSE)

$$
\begin{aligned}
R M S E_{c} & =\sqrt{\frac{1}{N} \sum_{i=1}^{N}\left(e_{x}^{2}(i)+e_{y}^{2}(i)+e_{z}^{2}(i)\right)} \\
R M S E_{r} & =\sqrt{\frac{1}{N} \sum_{i=1}^{N} e_{\alpha}^{2}(i)} \\
R M S E_{q} & =\sqrt{\frac{1}{N} \sum_{i=1}^{N} \sum_{j=1}^{4} e_{q_{j}}^{2}(i)}
\end{aligned}
$$

where $N$ is the number of recorded samples, $e_{x}, e_{y}, e_{z}$ denote the tracking error along the $x, y$ and $z$ axes, respectively, $e_{\alpha}$ is the tracking error of the platform's rotations and $e_{q_{i}}, i=1, \ldots, 4$ denotes the tracking error of the $\mathrm{i}^{\text {th }}$ actuator.

To compare the obtained performance in terms of energy consumption, the following input-torques-based criterion is proposed

$$
E_{\tau}=\sum_{i=1}^{4} \sum_{j=1}^{N}\left|\tau_{i}(j)\right|
$$

\section{A. Scenario 1: nominal case}

In this scenario, no additional payload is added to the moving platform. The motivation behind this scenario is to assess the adaptation capabilities of the proposed controller. To that aim, we consider that the mass of the moving platform as well as the arms' inertia are unknown. Thus, we initialize their values to zero and we let the estimation loop of the proposed controller estimate their steady-state values.

The Cartesian tracking errors of the moving platform of Veloce for this scenario are shown in Fig 3. For clarity 
_ TSM - - - Adaptive TSM
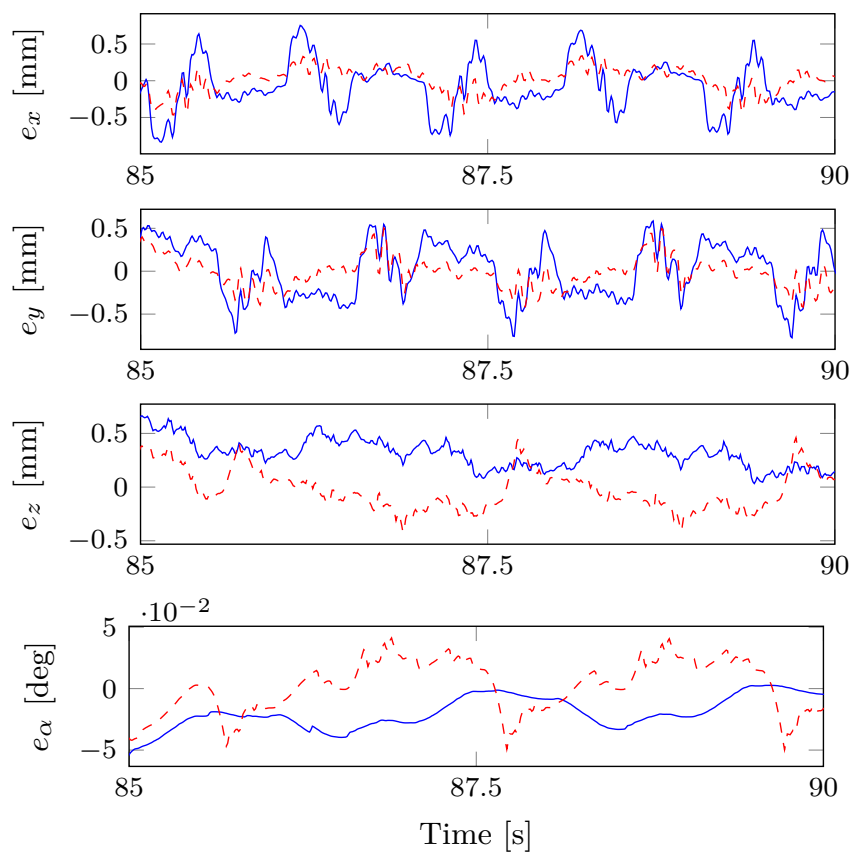

Fig. 3. Scenario 1: evolution of Cartesian tracking errors

of the view, the plots are zoomed around the interval $[85,90]$ s. As it can be seen, the tracking performance in the case of the proposed controller are significantly improved compared with TSM control.

The generated control input torques for both controllers are illustrated in Fig. 4. The plots are zoomed wihin the range $[85,90]$ seconds for clarity. We can see that the obtained control inputs for both controllers remain within allowable range. Based on the proposed performance-evaluation-criteria, the obtained results are summarized in Table III.

The time-evolution of the estimated moving platform and arms parameters is depicted in Fig. 5. We can notice that both parameters converge to their steadystate values within 20 seconds.

\section{B. Scenario 2: robustness to parameters variation}

Since the Veloce robot is intended for pick-and-place of lightweight objects, we consider in this scenario an additional payload attached to the moving platform of Veloce. The motivation of this scenario is to evaluate the

TABLE III

SCENARIO 1: PERFORMANCE EVALUATION OF BOTH CONTROLLERS

\begin{tabular}{|c|c|c|c|}
\hline & TSM & Adaptive TSM & Improvement \\
\hline$R M S E_{c}[\mathrm{~mm}]$ & 0.2096 & 0.0652 & $68.89 \%$ \\
$R M S E_{r}[\mathrm{deg}]$ & 0.0211 & 0.0173 & $18.01 \%$ \\
$R M S E_{j}[\mathrm{deg}]$ & 0.0190 & 0.0079 & $58.42 \%$ \\
$E_{\tau}[\mathrm{Nm}]$ & $4.2731 \times 10^{5}$ & $4.0539 \times 10^{5}$ & $5.13 \%$ \\
\hline
\end{tabular}

robustness of the proposed controller towards payload change. Similarly to the previous scenario, we initialize the mass of the moving platform and the inertia of the arms to zero. Then, the controller adjust their values in real-time in order to minimize the tracking errors.

The Cartesian tracking errors of the moving platform for this scenario are shown in Fig. 6. The plots are zoomed within the interval [85,90] s for clarity. Similar to the previous scenario, the proposed controller outperforms standard TSM controller in terms of tracking errors.

The generated control input torques are illustrated in Fig. 7. Also, in this scenario, the control torques remain within very safe margins. Based on the previously introduced error and torques based criteria, the performance of both controllers is summarized in Table IV.

The time-evolution of the estimated moving platform and arms parameters for the proposed adaptive controller is depicted in Fig 8. The estimated parameters, initialized to zero, converge to their steady-state values within 20 seconds.

TABLE IV

SCENARIO 2: PERFORMANCE EVALUATION OF BOTH CONTROLLERS

\begin{tabular}{|c|c|c|c|}
\hline & TSM & Adaptive TSM & Improvement \\
\hline$R M S E_{c}[\mathrm{~mm}]$ & 0.1640 & 0.0669 & $59.21 \%$ \\
$R M S E_{r}[\mathrm{deg}]$ & 0.0173 & 0.0158 & $9.49 \%$ \\
$R M S E_{j}[\mathrm{deg}]$ & 0.0173 & 0.0080 & $53.76 \%$ \\
$E_{\tau}[\mathrm{Nm}]$ & $4.2249 \times 10^{5}$ & $4.2139 \times 10^{5}$ & $0.26 \%$ \\
\hline
\end{tabular}



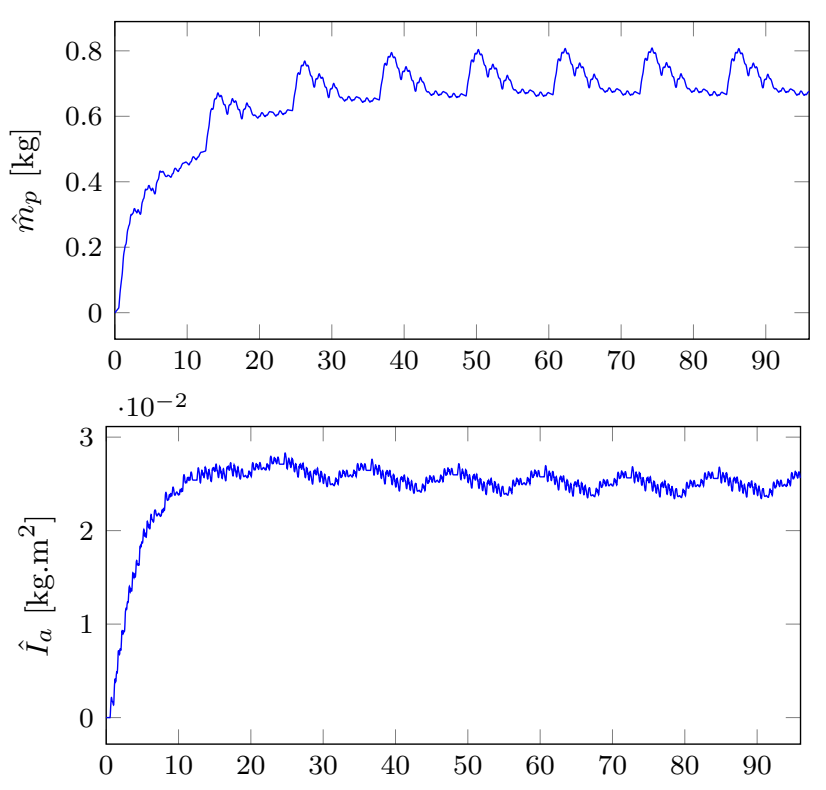

Fig. 5. Scenario 1: evolution of the estimated parameters.

\section{CONCLUSIONS AND FUTURE WORK}

A TSM-based adaptive controller for parallel manipulators has been proposed in this paper. TSM control is known for its robustness and high-performance thanks to its finite-time convergence property. In order to further improve the tracking capabilities of TSM control, we have proposed to extend it with an adaptive control loop based on the dynamic model of the system. The adapta-
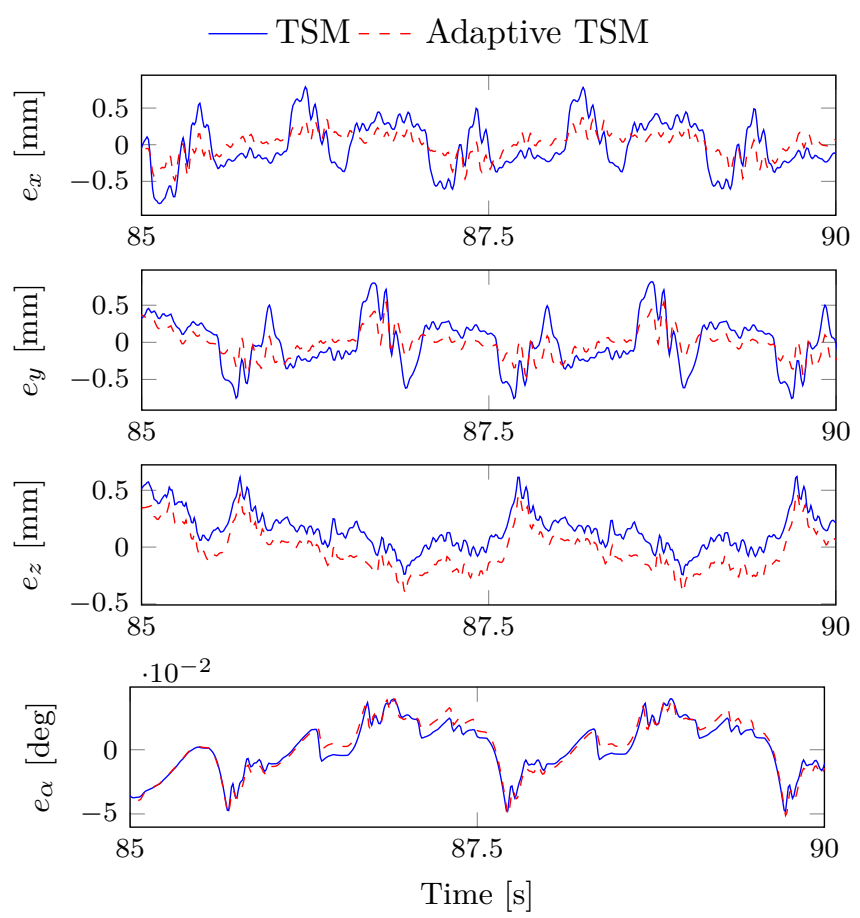

Fig. 6. Scenario 2: evolution of Cartesian tracking errors

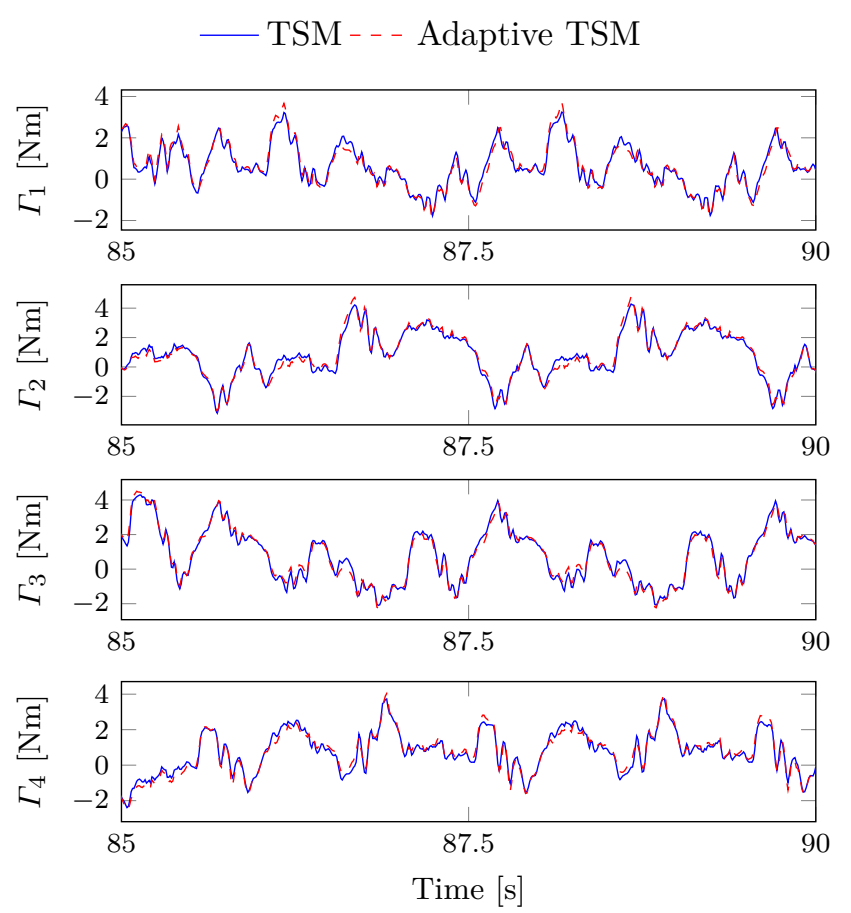

Fig. 7. Scenario 2: evolution of the control inputs

tion loop estimates in real-time the unknown, uncertain or time-varying parameters of the system in order for them to be used by the model-based control loop. The extended controller benefits from the advantages and features of both TSM and adaptive control. In order to evaluate the effectiveness of the proposed controller, realtime experiments were conducted on a 4-DOF parallel robot. The obtained results have shown improved track-
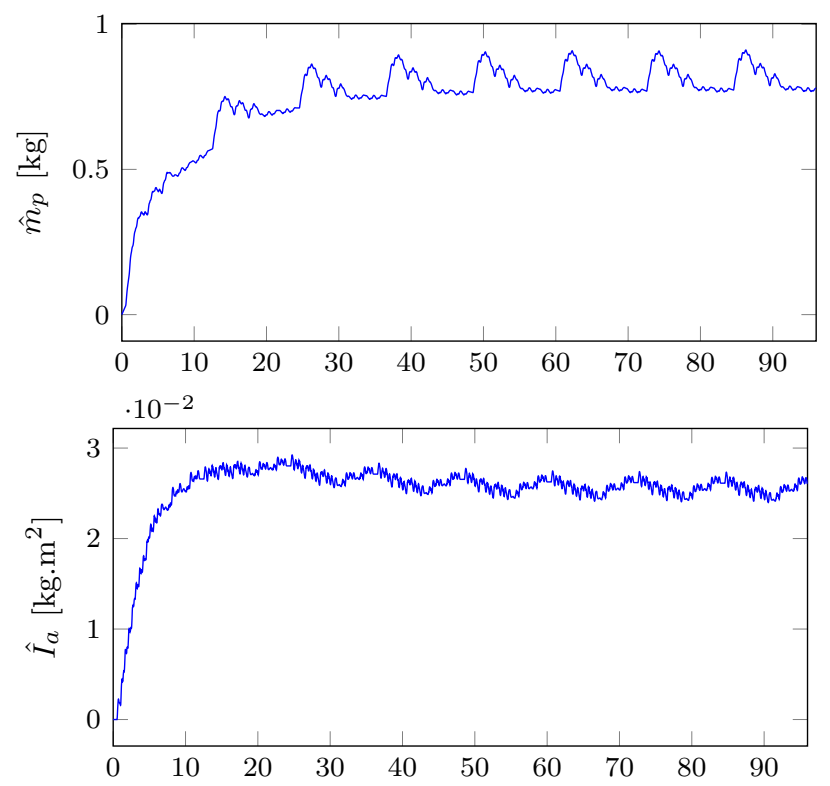

Fig. 8. Scenario 2: evolution of the estimated parameters. 
ing performance of the proposed controller.

\section{REFERENCES}

[1] J.-P. Merlet, Parallel Robots, Second Edition. Dordrecht, Netherlands: Springer, 2006.

[2] J.-P. Merlet and C. Gosselin, Springer Handbook of Robotics. Berlin, Heidelberg: Springer Berlin Heidelberg, 2008, ch. Parallel Mechanisms and Robots, pp. 269-285.

[3] S. Briot and W. Khalil, Dynamics of Parallel Robots: From Rigid Bodies to Flexible Elements. Springer, 2015, vol. 35.

[4] D. Zhang, Parallel Robotic Machine Tools, 1st ed. Springer US, 2010.

[5] V. Nabat, R. de la O, O. María, O. Company, S. Krut, and F. Pierrot, "Par4: very high speed parallel robot for pick-andplace," in Intelligent Robots and Systems, 2005.(IROS 2005). 2005 IEEE/RSJ International Conference on. IEEE, 2005, pp. $553-558$.

[6] N. Seward and I. A. Bonev, "A new 6-dof parallel robot with simple kinematic model," in Robotics and Automation (ICRA), 2014 IEEE International Conference on, May 2014, pp. 4061-4066.

[7] H. Saafi, M. A. Laribi, and S. Zeghloul, "Optimal haptic control of a redundant 3-rrr spherical parallel manipulator," in Intelligent Robots and Systems (IROS), 2015 IEEE/RSJ International Conference on. IEEE, 2015, pp. 2591-2596.

[8] L. Birglen, C. Gosselin, N. Pouliot, B. Monsarrat, and T. Laliberté, "SHaDe, A new 3-DOF haptic device," IEEE Transactions on Robotics and Automation, vol. 18, no. 2, pp. 166-175, 2002.

[9] W. Khalil and O. Ibrahim, "General solution for the dynamic modeling of parallel robots," Journal of Intelligent and Robotic Systems, vol. 49, no. 1, pp. 19-37, 2007.

[10] H. Cheng, Y. Yiu, and Z. Li, "Dynamics and control of redundantly actuated parallel manipulators," IEEE/ASME Transactions on Mechatronics, vol. 8, no. 4, pp. 483-491, Dec. 2003.

[11] F. Pierrot, C. Reynaud, and A. Fournier, "Delta: a simple and efficient parallel robot," Robotica, vol. 8, pp. 105-109, 41990.

[12] J. He, H. Jiang, D. Cong, and J. W. Ye, Z. M. an Han, "A survey on control of parallel manipulator," Key Engineering Materials, vol. 339, pp. 307-3013, May 2007.

[13] B. Siciliano, L. Sciavicco, L. Villani, and G. Oriolo, Robotics: modelling, planning and control. London, United Kingdom: Springer Science \& Business Media, 2009.
[14] J. W. F. Cheung and Y. S. Hung, "Modelling and control of a 2-dof planar parallel manipulator for semiconductor packaging systems," in Advanced Intelligent Mechatronics. Proceedings, 2005 IEEE/ASME International Conference on, July 2005, pp. $717-722$.

[15] Y. Zhang, S. Cong, W. Shang, Z. Li, and S. Jiang, "Modeling, identification and control of a redundant planar 2-dof parallel manipulator," International Journal of Control Automation and Systems, vol. 5, no. 5, pp. 559-569, 2007.

[16] J. J. Craig, P. Hsu, and S. S. Sastry, "Adaptive control of mechanical manipulators," The International Journal of Robotics Research, vol. 6, no. 2, pp. 16-28, 1987.

[17] W. Khalil and E. Dombre, Modeling, Identification and Control of Robots. London, United Kingdom: Kogan Page Science, 2004.

[18] J.-J. E. Slotine, W. Li et al., Applied nonlinear control. Prentice-Hall Englewood Cliffs, NJ, 1991, vol. 199, no. 1.

[19] M. Zhihong, A. Paplinski, and H. Wu, "A robust mimo terminal sliding mode control scheme for rigid robotic manipulators," Automatic Control, IEEE Transactions on, vol. 39, no. 12, pp. 2464-2469, Dec 1994.

[20] Y. Feng, X. Yu, and Z. Man, "Non-singular terminal sliding mode control of rigid manipulators," Automatica, vol. 38, no. 12, pp. 2159-2167, 2002.

[21] S. Yu, X. Yu, B. Shirinzadeh, and Z. Man, "Continuous finitetime control for robotic manipulators with terminal sliding mode," Automatica, vol. 41, no. 11, pp. 1957-1964, 2005.

[22] Y. Feng, X. Yu, and F. Han, "On nonsingular terminal slidingmode control of nonlinear systems," Automatica, vol. 49, no. 6, pp. $1715-1722,2013$.

[23] S. Mondal and C. Mahanta, "Adaptive second order terminal sliding mode controller for robotic manipulators," Journal of the Franklin Institute, vol. 351, no. 4, pp. 2356 - 2377, 2014.

[24] G. El-Ghazaly, M. Gouttefarde, and V. Creuze, Cable-Driven Parallel Robots: Proceedings of the Second International Conference on Cable-Driven Parallel Robots. Cham: Springer International Publishing, 2015, ch. Adaptive Terminal Sliding Mode Control of a Redundantly-Actuated Cable-Driven Parallel Manipulator: CoGiRo, pp. 179-200.

[25] R. Clavel, "Device for the movement and positioning of an element in space," Dec. 1985, uS Patent 4,976,582. [Online]. Available: https://patents.google.com/patent/US4976582A

[26] D. Corbel, M. Gouttefarde, and O. Company, "Towards $100 \mathrm{~g}$ with $\mathrm{pkm}$. is actuation redundancy a good solution for pick and place?" in Proc. IEEE International Conference on Robotics and Automation (ICRA'10), Anchorage, Alaska, May 2010, pp. 4675-4682. 